\title{
DNA copy number aberrations associated with the clinicopathological features of colorectal cancers: Identification of genomic biomarkers by array- based comparative genomic hybridization
}

\author{
MOTONAO NAKAO ${ }^{1}$, SHIGETO KAWAUCHI ${ }^{1}$, TETSUJI UCHIYAMA ${ }^{2}$, JUN ADACHI ${ }^{2}$, \\ HIDEAKI ITO $^{1}$, YASUYO CHOCHI ${ }^{1}$, TOMOKO FURUYA ${ }^{1}$, ATSUNORI OGA ${ }^{1}$ and KOHSUKE SASAKI ${ }^{1}$ \\ ${ }^{1}$ Department of Pathology, Yamaguchi University Graduate School of Medicine, Yamaguchi; \\ ${ }^{2}$ Department of Surgery, Iwakuni Medical Center of National Hospital Organization, Yamaguchi, Japan
}

Received December 28, 2010; Accepted February 24, 2011

DOI: 10.3892/or.2011.1246

\begin{abstract}
The aim of the present study was to investigate the chromosomal aberrations that are linked with the crucial clinicopathological features of colorectal cancer (CRC) and its prognosis by array-based comparative genomic hybridization (CGH). Fresh-frozen tumor tissues of 94 cases of CRC were analyzed by using bacterial artificial chromosome (BAC) CGH slides spotted with 4030 human BAC clones, which covered the whole range of the human genome at an average interval of 0.83 mega base pairs. DNA copy number aberrations (DCNAs) were identified in association with clinicopathological features: a gain of 8q24.3 and losses of 9q33.1 and 20p12.2 were associated with lymph node metastasis, gain of $8 \mathrm{q} 24.3$ and loss of 9q33.1 with disease stage, gain of $8 \mathrm{q} 21.11$ and loss of $10 \mathrm{q} 21.3$ with lymphovascular invasion and losses of 3p25.1, 10p15.3, 12q15 and 17p13.1 for venous invasion. These aberrations can be regarded as genomic biomarkers to predict the clinical outcome of patients with $\mathrm{CRC}$, and are expected to serve to individualize the treatment of CRC patients.
\end{abstract}

\section{Introduction}

Colorectal cancer (CRC) is one of the most common malignancies in humans worldwide, and apart from some familial types, usually arising sporadically (1). Cytogenetically, CRC can be classified into two types based on the types of genetic abnormalities present $(2,3)$. One is the major type, which is characterized by frequent chromosomal imbalances, that is,

Correspondence to: Dr Kohsuke Sasaki, Department of Pathology, Yamaguchi University Graduate School of Medicine, 1-1-1 Minamikogushi, Ube, Yamaguchi 755-8505, Japan

E-mail:kohsuke@yamaguchi-u.ac.jp

Key words: colorectal cancer, array-based comparative genome hybridization, DNA copy number aberration, genomic biomarker the chromosomal instability phenotype comprising more than $85 \%$ of all CRCs. The other is the minor type that frequently exhibits microsatellite instability originating from DNA replication errors. The microsatellite instability phenotype comprises $10-15 \%$ of all CRCs. In both types, the genomic instability leads to a degree of gains and losses of genomic DNA, which can be classified as DNA copy number aberrations (DCNAs).

Recently, microarray technology has been applied to the comparative genomic hybridization ( $\mathrm{CGH}$ ) methodology, thus leading to array-based comparative genomic hybridization (a-CGH) (4). The a-CGH method allows high-resolution and high-throughput screening of DCNAs across the whole genome. The DCNAs detected by the a-CGH method can be directly related to the DNA sequence information of a cancer to aid in the localization, identification and the validation of cancer-causing genes $(4,5)$.

In general, cancers (including CRC) occur as a result of the accumulation of a number of genomic aberrations that are linked with carcinogenesis and cancer progression $(2,3,6)$. Thus, the a-CGH analysis is suitable for studying the carcinogenic pathway of CRC with high-resolution and high-throughput results. Furthermore, the analysis of the cancer genome by a-CGH is expected to serve not only to clarify the relationship between the clinicopathological features and genomic abnormalities, but also to optimize the medical treatment of patients with CRC by making use of their cancer genome information. However, there has been little information available regarding the relationship between the tumor genome and its characteristics and the associated patient prognosis $(7,8)$.

The cytogenetic, as well as biological, properties of CRCs differ from each other. Thus, treatments based on the differences would be of benefit for the patients with CRC. Clinical information, such as the presence of lymph node metastasis and lymphovascular and blood vessel invasion, are predictors of prognosis and indicators of the optimal medical treatment approach (i.e. what kinds of adjuvant chemotherapy should be administered). The aim of the present study was to identify the 
Table I. Clinicopathological data.

\begin{tabular}{lc}
\hline Parameters & No. \\
\hline Gender & \\
Male & 51 \\
Female & 43 \\
UICC stage & \\
I + II & 38 \\
III + IV & 54 \\
Lymph node metastasis & \\
Negative & 41 \\
Positive & 43 \\
Lymphovascular invasion & \\
Negative & 54 \\
Positive & 31 \\
Venous invasion & \\
Negative & 36 \\
Positive & 50 \\
\hline
\end{tabular}

DCNAs that are linked with these crucial clinicopathological features of CRCs and to provide the genomic information that can be valuable for the treatment of patients with CRC.

\section{Materials and methods}

Materials. The present study followed the ethical guidelines of the Institutional Review Board of the Yamaguchi University School of Medicine. Ninety-four surgically resected, freshfrozen CRC sample tissues were available for the present study. The patients consisted of 51 males and 43 females, ranging from 39 to 87 years in age (Table I). The clinical staging of the tumors was according to the UICC TNM Classification, 2002 (9). Histopathologically, 63 of the tumors were diagnosed as well differentiated, 25 as moderately differentiated, and 6 as poorly differentiated adenocarcinomas.

Array-based comparative genomic hybridization. Tumor tissue sections (10-mm thick) were cut from each fresh-frozen cancer tissue specimen using a cryostat (Bright Instrument, Hunchington, UK). The sections were immediately fixed in 90\% ethanol solution and stained with methylgreen (Sigma, Tokyo, Japan). Tumor tissues were microdissected using a 28-gauge needle. High molecular weight genomic DNA was extracted from the cancer tissue sections using a DNeasy Tissue kit (Qiagen Sciences, CA, USA).

Each $500 \mathrm{ng}$ tumor DNA sample and reference DNA sample were labeled with FluoroLink Cy5-dCTP (PerkinElmer, MA, USA) and FluoroLink Cy3-dCTP (Perkin-Elmer) using a BioPrime DNA Labeling System (Promega, WI, USA), respectively. The fluorescence-labeled DNAs were applied to a MacArray Karyo4000 CGH array slide (Macrogen, Seoul, Korea). The array slide was spotted with 4030 human bacterial artificial chromosome (BAC) clones, which covered the whole range of the human genome at an average interval of 0.83 mega base pairs $(\mathrm{Mb})$. Images of the 16-bit fluorescence intensity for spots were captured using a GenePix 4000A

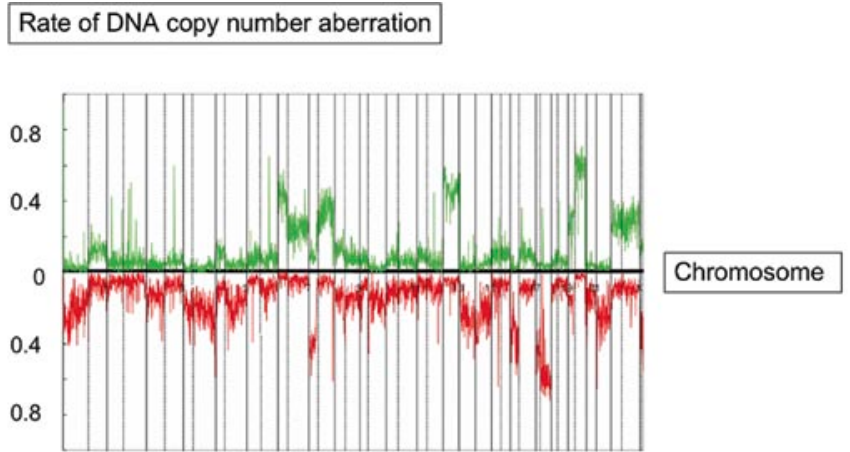

Figure 1. Overall frequency of DNA copy number aberrations detected by a-CGH for each BAC clone in 94 colorectal adenocarcinomas. The frequency of aberrations is depicted as the fraction of cases with DNA copy number gain or loss for the $4030 \mathrm{BAC}$ clones (the entire genome). The green dots in the upper part of the profile indicate the frequency of tumors with DNA copy number gains, and the red dots in the lower part of the profile indicate the frequency of tumors with DNA copy number losses.

Table II. Top 10 loci associated with DNA copy number aberrations in 94 colorectal cancers.

\begin{tabular}{lc}
\hline Locus & Frequency $(\%)$ \\
\hline DNA copy number gain & \\
20q11 & 76 \\
$20 q 13$ & 71 \\
$20 q 12$ & 68 \\
$13 q 12$ & 61 \\
$13 q 34$ & 58 \\
$7 p 14$ & 58 \\
$13 q 22$ & 56 \\
$13 q 13$ & 56 \\
$7 p 21$ & 55 \\
$13 q 33$ & 54 \\
DNA copy number loss & \\
$18 q 23$ & 74 \\
$18 q 21$ & 71 \\
$18 q 12$ & 70 \\
$22 q 11$ & 67 \\
$18 q 22$ & 67 \\
$18 q 23$ & 66 \\
$17 p 11$ & 63 \\
$18 q 22$ & 63 \\
$4 q 35$ & 61 \\
$17 p 13$ & 60 \\
\hline
\end{tabular}

scanner (Axon Instruments, CA, USA), and the Cy5/Cy3 ratio values were calculated using the MAC Viewer software program (Macrogen). All fluorescence intensity ratios were converted to $\log$ base 2 . Any inadequate spots were flagged by manual inspection.

Data analysis. The $\chi^{2}$ test was used to identify the DCNAs by analyzing a-CGH data of CRC with and without nodal metastasis, lymphatic invasion, venous invasion, and highand low-clinical stage. Figures were generated to determine 
A

$$
p
$$

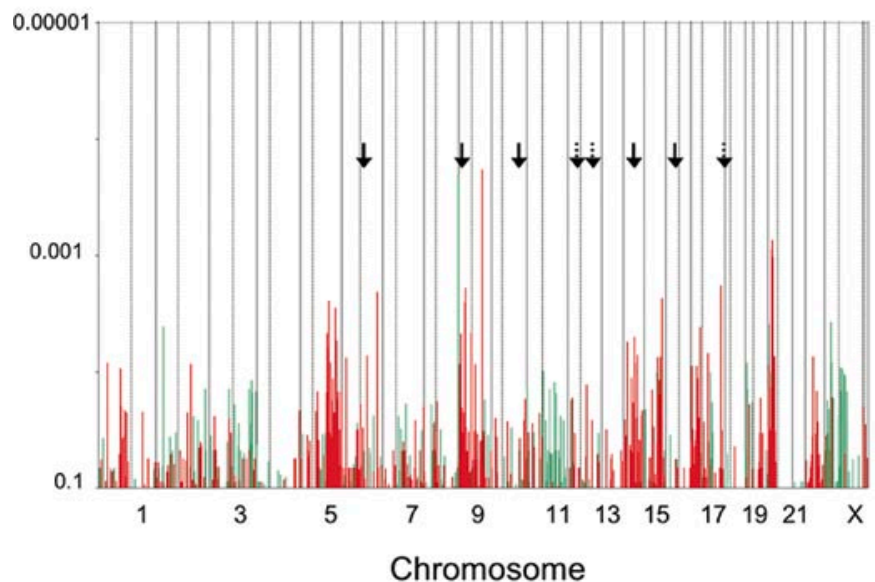

C

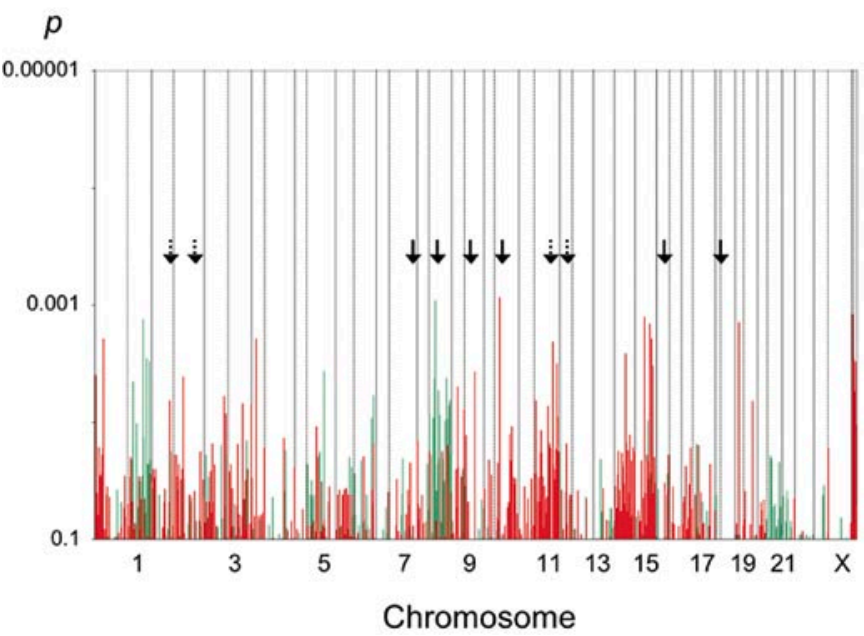

B

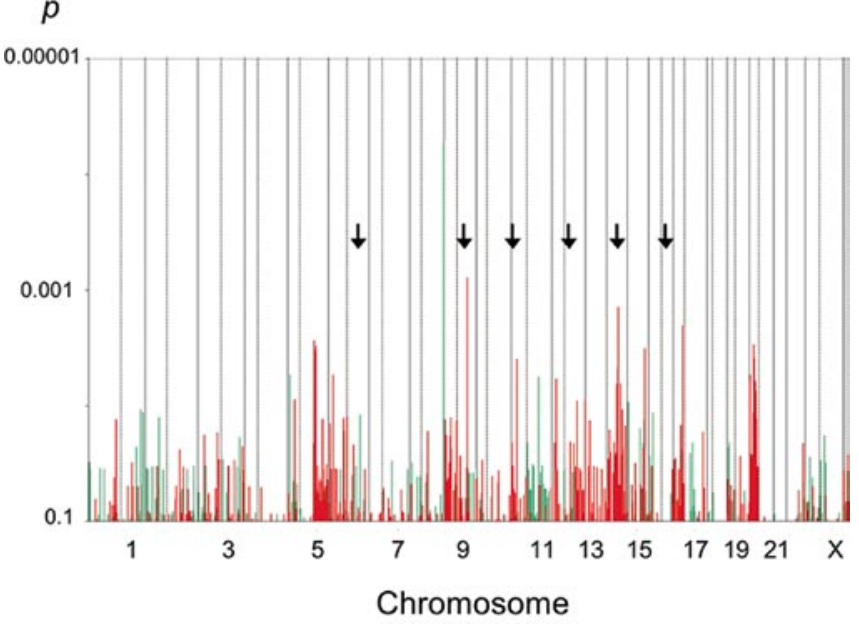

D

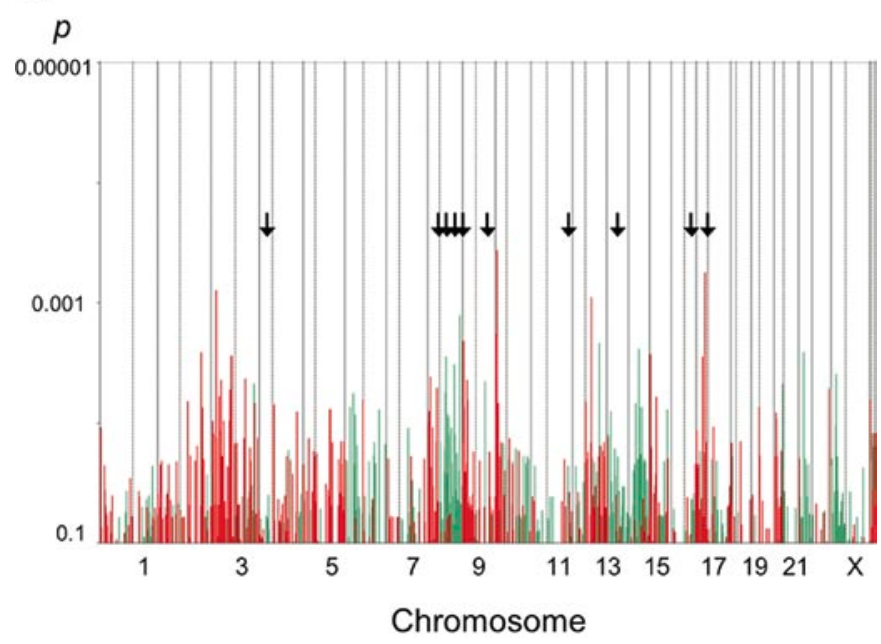

Figure 2. The results of the $\chi^{2}$ test for the 4030 BAC clones and the differences in the p-values between lymph node metastasis negative and positive (A), low stage (stages I and II) and advanced stage (stages III and IV) (B), lymphovascular invasion negative and positive (C), and venous invasion negative and positive (D) colorectal cancers (CRCs). The green columns of each profile indicate an increase in the frequency of CRCs carrying DNA copy number aberrations (DCNAs) at the corresponding chromosome locus in the former categories, ie, lymph node metastasis negative (A), low stage (B), lymphovascular invasion negative (C), or venous invasion negative (D) categories. The red columns of each profile indicate the increase in frequency of CRCs carrying DCNAs at the corresponding chromosomal locus in the same categories (lymph node metastasis positive (A), high stage (B), lymphovascular invasion positive (C), or venous invasion positive (D) categories).

the difference by plotting the BAC DNA clones with $\mathrm{p}<0.1$ based on the analyses (Figs. 1 and 2).

\section{Results}

The BAC clones indicated that the DCNAs differed for every case. Although there were differences in the DCNAs between each of the 94 cases of CRC, the most frequent DCN gain was of $20 q 11$, which was observed in $76 \%$ of the cases. The frequency of DNA copy number gain at $13 q$ and $7 p(58 \%)$ was also high (Table II). On the other hand, the most frequent DNA copy number loss was at $18 \mathrm{q} 23$, and was detected in $74 \%$ of the cases (Table II).

Lymph node metastasis and DCNA. Fig. 2A shows the results of the $\chi^{2}$ test between CRCs with lymph node metastasis and those without metastasis with regard to all 4030 of the BAC clones mounted on a-CGH slide, as well as the corresponding p-values. Fig. 2A shows that the CRCs with lymph node metastasis were associated with a gain of 11q and losses of $5 q$, 9, 17p and 20p (arrows, $\mathrm{p}<0.01$ ). The figure also demonstrates that CRCs without lymph node metastasis were associated with a gain of $\mathrm{X}$, and losses of chromosomes 14 and 15 (dotted arrows, $\mathrm{p}<0.01)$.

The specific BAC clones with $\mathrm{p}<0.01$ are listed in Table III. CRCs with lymph node metastasis were significantly associated with a gain of clone 2748 (8q24.3) and losses of clone 4289 (9q33.1), clone 5951 (20p12.2) and clone 466 (20p12.2) $(\mathrm{p}<0.001)$.

Clinical stage and DCNA. CRCs in stages I and II were defined as low stage tumors, and those in stages III and IV as advanced stage tumors. Fig. $2 \mathrm{~B}$ shows the results of the $\chi^{2}$ test between low stage CRCs and advanced stage CRCs 
Table III. BAC clones associated with lymph node metastasis in colorectal cancer.

\begin{tabular}{|c|c|c|c|c|c|}
\hline Chromosome & BAC-start & Locus & ID & Locating genes & P-value \\
\hline \multicolumn{6}{|c|}{ DNA copy number gain } \\
\hline 2 & 20378550 & $2 \mathrm{p} 24.1$ & 1357 & PUM2 & 0.004108 \\
\hline 8 & $1.46 \mathrm{E}+08$ & $8 \mathrm{q} 24.3$ & 2748 & ZNF16, TMED10P, C8orf77 & 0.000203 \\
\hline 10 & 25715182 & Xp21.3 & 4329 & & 0.003777 \\
\hline 10 & 29992424 & $\mathrm{Xp} 21.2$ & 2150 & MAGEB2, MAGEB3, MAGEB4, MAGEB1, NR0B1 & 0.008468 \\
\hline 10 & 70247389 & Xq13.1 & 2922 & ZMYM3, NONO, ITGB1BP2 & 0.009193 \\
\hline 10 & 74789201 & Xq13.3 & 5171 & & 0.009406 \\
\hline 11 & 60762733 & $11 \mathrm{q} 12.2$ & 2373 & PGA5, VWCE, DDB1 & 0.009995 \\
\hline 15 & 84084157 & $15 q 25.3$ & 2914 & AKAP13, KLHL25 & 0.00766 \\
\hline 19 & 858881 & $19 \mathrm{p} 13.3$ & 5529 & C19orf22, KISS1R, ARID3A, WDR18, GRIN3B, C19orf6, ABCA7 & 0.008422 \\
\hline 20 & 705254 & $20 \mathrm{p} 13$ & 5734 & C20orf55, RPS10L, ANGPT4 & 0.003907 \\
\hline 20 & 14725959 & 20p12.1 & 1089 & C20orf133 & 0.007449 \\
\hline \multicolumn{6}{|c|}{ DNA copy number loss } \\
\hline 1 & 28059305 & $1 \mathrm{p} 35.3$ & 922 & EYA3 & 0.008444 \\
\hline 1 & 76485020 & $1 \mathrm{p} 31.1$ & 4085 & ST6GALNAC3 & 0.009498 \\
\hline 2 & $1.62 \mathrm{E}+08$ & $2 \mathrm{q} 24.2$ & 5373 & PSMD14, TBR1 & 0.008638 \\
\hline 5 & $1.32 \mathrm{E}+08$ & $5 \mathrm{q} 23.3$ & 5300 & P4HA2, PDLIM4, SLC22A4 & 0.002469 \\
\hline 5 & $1.52 \mathrm{E}+08$ & $5 q 33.1$ & 4740 & & 0.002835 \\
\hline 5 & $1.25 \mathrm{E}+08$ & $5 \mathrm{q} 23.2$ & 4761 & & 0.004705 \\
\hline 5 & $1.51 \mathrm{E}+08$ & $5 q 33.1$ & 5345 & SPARC, ATOX1 & 0.005411 \\
\hline 5 & $1.59 \mathrm{E}+08$ & $5 q 33.3$ & 2853 & & 0.005413 \\
\hline 5 & $1.26 \mathrm{E}+08$ & $5 q 23.2$ & 4736 & LMNB1, MARCH3 & 0.006226 \\
\hline 5 & $1.37 \mathrm{E}+08$ & $5 q 31.1$ & 4668 & SPOCK1 & 0.008393 \\
\hline 5 & $1.35 \mathrm{E}+08$ & $5 q 31.1$ & 1539 & TGFBI, SMAD5 & 0.009836 \\
\hline 6 & $1.52 \mathrm{E}+08$ & $6 q 25.1$ & 173 & ESR1, SYNE1 & 0.002059 \\
\hline 6 & 98369873 & $6 q 16.1$ & 4205 & & 0.007345 \\
\hline 6 & 14035344 & $6 \mathrm{p} 23$ & 814 & RNF182 & 0.007675 \\
\hline 9 & $1.17 \mathrm{E}+08$ & $9 \mathrm{q} 33.1$ & 4289 & & 0.000183 \\
\hline 9 & 23737636 & $9 \mathrm{p} 21.3$ & 1099 & ELAVL2 & 0.001923 \\
\hline 9 & 21726045 & $9 \mathrm{p} 21.3$ & 4214 & MTAP & 0.002536 \\
\hline 9 & $1.17 \mathrm{E}+08$ & $9 q 33.1$ & 4221 & ASTN2, TRIM32 & 0.003014 \\
\hline 9 & 42141245 & $9 \mathrm{p} 12$ & 5118 & & 0.004607 \\
\hline 9 & 919127 & $9 \mathrm{p} 24.3$ & 5884 & DMRT1, DMRT3 & 0.004775 \\
\hline 9 & 414664 & $9 \mathrm{p} 24.3$ & 5566 & DOCK8, ANKRD15 & 0.008638 \\
\hline 9 & 79353236 & $9 q 21.31$ & 1119 & TLE4 & 0.008638 \\
\hline 14 & 71175222 & $14 q 24.2$ & 4675 & SIPA1L1 & 0.005054 \\
\hline 14 & 34875953 & $14 q 13.2$ & 2531 & NFKBIA & 0.005595 \\
\hline 14 & 80102060 & $14 q 31.1$ & 1280 & C14orf145 & 0.007222 \\
\hline 14 & 73441348 & $14 q 24.3$ & 4034 & ZNF410, C14orf44, COQ6, ENTPD5 & 0.008437 \\
\hline 15 & 89157227 & $15 q 26.1$ & 2434 & BLM, FURIN, FES, MAN2A2 & 0.002363 \\
\hline 15 & 89185022 & $15 q 26.1$ & 2971 & FURIN, FES, MAN2A2, HDDC3 & 0.003354 \\
\hline 15 & 88125742 & $15 q 26.1$ & 707 & ANPEP, AP3S2 & 0.007458 \\
\hline 15 & 71713010 & $15 \mathrm{q} 24.1$ & 4618 & $\mathrm{CD} 276$ & 0.007576 \\
\hline 17 & 72955036 & $17 q 25.3$ & 2602 & SEPT9 & 0.001816 \\
\hline 17 & 11944544 & $17 \mathrm{p} 12$ & 2859 & MAP2K4 & 0.004177 \\
\hline 17 & 35871401 & $17 q 21.2$ & 2983 & TNS4 & 0.006952 \\
\hline 17 & 2510194 & $17 \mathrm{p} 13.3$ & 5917 & PAFAH1B1, KIAA0664 & 0.009045 \\
\hline 17 & 410041 & $17 \mathrm{p} 13.3$ & 158 & VPS53 & 0.009053 \\
\hline 20 & 10407110 & 20p12.2 & 5951 & C20orf94 & 0.000748 \\
\hline 20 & 9192646 & 20p12.2 & 466 & PLCB4 & 0.000894 \\
\hline 20 & 10566034 & $20 \mathrm{p} 12.2$ & 768 & JAG1 & 0.001033 \\
\hline 20 & 10633685 & $20 \mathrm{p} 12.2$ & 5954 & & 0.005326 \\
\hline 20 & 10266917 & $20 \mathrm{p} 12.2$ & 5952 & RPL23AP6, MKKS, C20orf94 & 0.00536 \\
\hline 20 & 10740672 & $20 \mathrm{p} 12.2$ & 5957 & FAT1P1 & 0.007335 \\
\hline 20 & 16603508 & $20 \mathrm{p} 12.1$ & 1509 & RPL7AL3, SNRPB2, OTOR & 0.007452 \\
\hline 20 & 12524678 & $20 \mathrm{p} 12.1$ & 1345 & & 0.008638 \\
\hline 20 & 168339 & $20 \mathrm{p} 13$ & 5621 & C20orf96, ZCCHC3, SOX12, C20orf98, TRIB3 & 0.008801 \\
\hline 22 & 27639138 & $22 q 12.1$ & 460 & ZNRF3 & 0.007449 \\
\hline
\end{tabular}


Table IV. BAC clones associated with clinical stage in colorectal cancer.

\begin{tabular}{|c|c|c|c|c|c|}
\hline Chromosome & BAC-start & Locus & ID & Locating genes & P-value \\
\hline \multicolumn{6}{|l|}{$\begin{array}{l}\text { DNA copy } \\
\text { number gain }\end{array}$} \\
\hline 5 & 886825 & $5 p 15.33$ & 5598 & ZDHHC11, BRD9, TRIP13 & 0.005423 \\
\hline 8 & $1.46 \mathrm{E}+08$ & $8 q 24.3$ & 2748 & ZNF16, TMED10P, C8orf77 & $5.14 \mathrm{E}-05$ \\
\hline 11 & 94501857 & $11 q 21$ & 1302 & ENDOD1, SESN3 & 0.00567 \\
\hline 15 & 19958051 & $15 \mathrm{q} 11.2$ & 2266 & VSIG6 & 0.009342 \\
\hline \multicolumn{6}{|l|}{$\begin{array}{l}\text { DNA copy } \\
\text { number loss }\end{array}$} \\
\hline 5 & $1.25 \mathrm{E}+08$ & $5 q 23.2$ & 4761 & & 0.00273 \\
\hline 5 & $1.32 \mathrm{E}+08$ & $5 q 23.3$ & 5300 & P4HA2, PDLIM4, SLC22A4 & 0.003043 \\
\hline 5 & $1.26 \mathrm{E}+08$ & $5 q 23.2$ & 4736 & LMNB1, MARCH3 & 0.003359 \\
\hline 5 & 18102403 & $5 \mathrm{p} 15.1$ & 4466 & & 0.00887 \\
\hline 6 & 14035344 & $6 \mathrm{p} 23$ & 814 & RNF182 & 0.005457 \\
\hline 9 & $1.17 \mathrm{E}+08$ & $9 q 33.1$ & 4289 & & 0.00078 \\
\hline 9 & $1.17 \mathrm{E}+08$ & $9 q 33.1$ & 4221 & ASTN2, TRIM32 & 0.008174 \\
\hline 11 & 8182603 & $11 \mathrm{p} 15.4$ & 2704 & LMO1 & 0.003937 \\
\hline 12 & 6175040 & $12 \mathrm{p} 13.31$ & 591 & CD9 & 0.005891 \\
\hline 12 & $1.32 \mathrm{E}+08$ & $12 \mathrm{q} 24.33$ & 5605 & ZNF26, ZNF84, ZNF140 & 0.008955 \\
\hline 12 & 96497364 & $12 \mathrm{q} 23.1$ & 4796 & & 0.009111 \\
\hline 14 & 73441348 & $14 \mathrm{q} 24.3$ & 4034 & ZNF410, C14orf44, COQ6, ENTPD5 & 0.001408 \\
\hline 14 & 71175222 & $14 \mathrm{q} 24.2$ & 4675 & SIPA1L1 & 0.004751 \\
\hline 14 & 80102060 & $14 \mathrm{q} 31.1$ & 1280 & C14orf145 & 0.006465 \\
\hline 14 & 63739020 & $14 \mathrm{q} 23.2$ & 2040 & SYNE2, ESR2 & 0.006543 \\
\hline 15 & 89157227 & $15 \mathrm{q} 26.1$ & 2434 & BLM, FURIN, FES, MAN2A2 & 0.003217 \\
\hline 17 & 11944544 & $17 \mathrm{p} 12$ & 2859 & MAP2K4 & 0.002045 \\
\hline 20 & 10407110 & $20 \mathrm{p} 12.2$ & 5951 & C20orf94 & 0.002978 \\
\hline 20 & 10566034 & $20 \mathrm{p} 12.2$ & 768 & JAG1 & 0.003914 \\
\hline 20 & 513026 & 20 p13 & 5646 & TCF15, SRXN1, SCRT2 & 0.005423 \\
\hline 20 & 168339 & $20 \mathrm{p} 13$ & 5621 & C20orf96, ZCCHC3, SOX12, C20orf98, TRIB3 & 0.006151 \\
\hline 20 & 15275392 & $20 \mathrm{p} 12.1$ & 269 & C20orf133 & 0.006172 \\
\hline 20 & 16603508 & $20 \mathrm{p} 12.1$ & 1509 & RPL7AL3, SNRPB2, OTOR & 0.007495 \\
\hline
\end{tabular}

for all 4030 BAC clones mounted on the a-CGH slides with the corresponding p-values. The figure also shows that the advanced stage CRCs were associated with a gain of 11q and losses of 5q, 9, 14, 17p and 20p (arrows, p<0.01).

The specific clones with $\mathrm{p}<0.01$ are listed in Table IV. Advanced stage CRCs were significantly associated with a gain of clone 2748 (8q24.3) and a loss of clone 4289 (9q33.1) $(\mathrm{p}<0.001)$. These two BAC clones that were associated with advanced stage CRCs were also included in those that were linked with CRCs with lymph node metastasis.

Lymphovascular invasion and DCNA. Fig. 2C shows the p-values corresponding to the $4030 \mathrm{BAC}$ clones that were estimated by the $\chi^{2}$ test between CRCs with lymphovascular invasion and those without lymphovascular invasion. The CRCs with lymphovascular invasion were associated with an increased frequency of gains of $8 \mathrm{q}$ and 21 , and losses of 10, 11 and $\mathrm{Y}$ (arrows, $\mathrm{p}<0.01$ ). Further, Fig. $2 \mathrm{C}$ shows that the CRCs without lymphovascular invasion were associated with a gain of 1q, and losses of 1p, 14 and 15p (dotted arrows, $\mathrm{p}<0.01)$.
The specific BAC clones with $\mathrm{p}<0.01$ are listed in Table V. CRCs with lymphovascular invasion were significantly associated with a gain of clone $4208(8 \mathrm{q} 21.11)$ and a loss of clone 4467 (10q21.3) $(\mathrm{p}<0.001)$.

Venous invasion and DCNA. Fig. 2D shows the results of the $\chi^{2}$ test between CRCs with venous invasion and those without venous invasion concerning all 4030 of the BAC clones mounted on a-CGH slides with their corresponding p-values. The CRCs free of venous invasion were associated with gains of 8q, 14, 22 and Xp, and losses of 3p, 8p, 9p, 10p and $17 \mathrm{p}$ (arrows, $\mathrm{p}<0.01$ ).

The specific BAC clones with $\mathrm{p}<0.01$ are listed in Table VI. CRCs with venous invasion were significantly associated with losses of clone 230 (10p15.3), 1536 (17p13.1), 2562 (3p25.1) and clone $2084(12 \mathrm{q} 15)(\mathrm{p}<0.001)$.

\section{Discussion}

The biological properties of cancers differ by patient and by cancer subtype. Thus, treatments based on an individual cancer would be of benefit to patients. Clinically significant 
Table V. BAC clones with lymphovascular invasion in colorectal cancer.

\begin{tabular}{|c|c|c|c|c|c|}
\hline Chromosome & BAC-start & Locus & ID & Locating genes & P-value \\
\hline \multicolumn{6}{|c|}{ DNA copy number gain } \\
\hline 1 & 208000000 & $1 \mathrm{q} 32.3$ & 1078 & SLC30A1, NEK2 & 0.001329 \\
\hline 1 & 229000000 & $1 \mathrm{q} 42.2$ & 5630 & SIPA1L2 & 0.002892 \\
\hline 1 & 242000000 & $1 q 44$ & 303 & & 0.003034 \\
\hline 1 & 157000000 & $1 \mathrm{q} 23.2$ & 1412 & PIGM, KCNJ10 & 0.004509 \\
\hline 5 & 135000000 & $5 q 31.1$ & 2688 & IL9, FBXL21, LECT2 & 0.003663 \\
\hline 6 & 168000000 & $6 q 27$ & 2706 & MLLT4 & 0.005968 \\
\hline 6 & 160000000 & $6 q 25.3$ & 2872 & SOD2, WTAP & 0.009212 \\
\hline 8 & 75824814 & $8 \mathrm{q} 21.11$ & 4208 & PI15 & 0.00092 \\
\hline 8 & 134000000 & $8 \mathrm{q} 24.22$ & 359 & WISP1, NDRG1 & 0.004182 \\
\hline 8 & 72080962 & $8 \mathrm{q} 13.3$ & 5059 & & 0.004275 \\
\hline 8 & 91413409 & $8 q 21.3$ & 1131 & & 0.005384 \\
\hline 8 & 145000000 & $8 q 24.3$ & 5267 & HSF1, DGAT1, SCRT1, FBXL6, GPR172A, ADCK5, CPSF1 & 0.00651 \\
\hline 8 & 145000000 & $8 \mathrm{q} 24.3$ & 1284 & ZC3H3, GSDMDC1, C8orf73, NAPRT1, EEF1D & 0.007132 \\
\hline 8 & 48721528 & $8 \mathrm{q} 11.21$ & 2413 & CEBPD & 0.008533 \\
\hline 8 & 99509595 & $8 \mathrm{q} 22.2$ & 4470 & KCNS2, STK3 & 0.008827 \\
\hline 8 & 66247296 & $8 q 13.1$ & 4923 & & 0.009245 \\
\hline 8 & 143000000 & $8 \mathrm{q} 24.3$ & 4223 & & 0.009392 \\
\hline 8 & 128000000 & $8 \mathrm{q} 24.21$ & 939 & SRRM1L & 0.009718 \\
\hline 15 & 70609648 & $15 q 24.1$ & 334 & ARIH1 & 0.009665 \\
\hline \multicolumn{6}{|c|}{ DNA copy number loss } \\
\hline 1 & 28068722 & $1 \mathrm{p} 35.3$ & 388 & EYA3 & 0.001934 \\
\hline 1 & 1463003 & $1 \mathrm{p} 36.33$ & 705 & SLC35E2, CDC2L2, CDC2L1 & 0.00402 \\
\hline 2 & 140000000 & $2 q 22.1$ & 4557 & & 0.004082 \\
\hline 2 & 68918173 & $2 \mathrm{p} 13.3$ & 5007 & ARHGAP25 & 0.00654 \\
\hline 3 & 71826780 & $3 \mathrm{p} 13$ & 900 & EIF4E3, GPR27, PROK2 & 0.005996 \\
\hline 3 & 166000000 & $3 q 26.1$ & 4469 & SLITRK3 & 0.007001 \\
\hline 3 & 199000000 & $3 q 29$ & 2321 & KIAA0226 & 0.00714 \\
\hline 3 & 80273927 & $3 \mathrm{p} 12.2$ & 4988 & & 0.00844 \\
\hline 3 & 167000000 & $3 q 26.1$ & 4805 & & 0.009354 \\
\hline 4 & 3180037 & $4 \mathrm{p} 16.3$ & 2855 & HD & 0.001934 \\
\hline 9 & 117000000 & $9 q 33.1$ & 4289 & & 0.003724 \\
\hline 9 & 21726045 & $9 \mathrm{p} 21.3$ & 4214 & MTAP & 0.004983 \\
\hline 9 & 42141245 & $9 \mathrm{p} 12$ & 5118 & & 0.00783 \\
\hline 10 & 67611287 & $10 \mathrm{q} 21.3$ & 4467 & CTNNA3 & 0.000866 \\
\hline 11 & 128000000 & $11 \mathrm{q} 24.3$ & 1184 & FLI1 & 0.003167 \\
\hline 11 & 61848708 & $11 \mathrm{q} 12.3$ & 2681 & ASRGL1, SCGB1A1, AHNAK & 0.00654 \\
\hline 11 & 101000000 & $11 q 22.1$ & 35 & & 0.007308 \\
\hline 11 & 131000000 & $11 \mathrm{q} 25$ & 4032 & C11orf39 & 0.009046 \\
\hline 14 & 71175222 & $14 \mathrm{q} 24.2$ & 4675 & SIPA1L1 & 0.002616 \\
\hline 15 & 48316625 & $15 \mathrm{q} 21.2$ & 985 & HDC, GABPB2 & 0.001262 \\
\hline 15 & 76950406 & $15 \mathrm{q} 25.1$ & 4450 & MORF4L1, CTSH, RASGRF1 & 0.001444 \\
\hline 15 & 88125742 & $15 q 26.1$ & 707 & ANPEP, AP3S2 & 0.001934 \\
\hline 15 & 89185022 & $15 q 26.1$ & 2971 & FURIN, FES, MAN2A2, HDDC3 & 0.00332 \\
\hline 15 & 49208466 & $15 \mathrm{q} 21.2$ & 590 & CYP19A1 & 0.007401 \\
\hline 19 & 10175587 & $19 \mathrm{p} 13.2$ & 753 & EDG5, ICAM1, ICAM4 & 0.001418 \\
\hline 19 & 54042891 & $19 q 13.33$ & 1194 & PLEKHA4, PPP1R15A, TULP2, NUCB1, DHDH, BAX, FTL, GYS1 & 0.006621 \\
\hline $\mathrm{Y}$ & 6062912 & Yp11.2 & 4648 & TSPY2, TSPYP1 & 0.001209 \\
\hline $\mathrm{Y}$ & 10145611 & Yp11.2 & 24 & RBMY2GP, TTTY7 & 0.002883 \\
\hline Y & 21750616 & Yq11.223 & 899 & RBMY2SP & 0.00305 \\
\hline Y & 21831283 & Yq11.223 & 485 & RBMY2EP, RBMY2TP, TSPYP4 & 0.003814 \\
\hline $\mathrm{Y}$ & 19261015 & Yq11.222 & 1442 & USP9YP1, HSFY1, TTTY9B, OFDYP5 & 0.005537 \\
\hline Y & 18634292 & Yq11.221 & 412 & CDY5P, ACTGP2, XKRY, SEDLP3, OFDYP1 & 0.005716 \\
\hline $\mathrm{Y}$ & 6245999 & Yp11.2 & 5143 & RBMY2GP, TTTY7 & 0.00592 \\
\hline Y & 6123032 & Yp11.2 & 4330 & TSPY2, TSPYP1, RBMY2GP & 0.006672 \\
\hline Y & 22359301 & Yq11.223 & 429 & RBMY1B, RBMY1A1 & 0.007153 \\
\hline $\mathrm{Y}$ & 13424058 & Yq11.21 & 4549 & DDX3Y, CASKP & 0.008572 \\
\hline
\end{tabular}


Table VI. BAC clones associated with venous invasion in colorectal cancer.

\begin{tabular}{|c|c|c|c|c|c|}
\hline Chromosome & BAC-start & Locus & ID & Locating genes & P-value \\
\hline \multicolumn{6}{|l|}{$\begin{array}{l}\text { DNA copy } \\
\text { number gain }\end{array}$} \\
\hline 3 & $1.85 \mathrm{E}+08$ & $3 q 27.1$ & 1267 & AP2M1, ABCF3, ALG3, CAMK2N2 & 0.004777 \\
\hline 6 & 31614370 & $6 \mathrm{p} 21.33$ & 2279 & $\begin{array}{l}\text { BAT1, ATP6V1G2, NFKBIL1, LTA, TNF, LTB, LST1, } \\
\text { NCR3, AIF1, BAT2, BAT3 }\end{array}$ & 0.005702 \\
\hline 6 & 19479260 & $6 \mathrm{p} 22.3$ & 4509 & & 0.007369 \\
\hline 6 & $1.48 \mathrm{E}+08$ & $6 \mathrm{q} 24.3$ & 838 & & 0.007798 \\
\hline 6 & 32917633 & $6 \mathrm{p} 21.32$ & 2086 & PSMB8, TAP1, PSMB9, PPP1R2P1, HLA-DMB & 0.008609 \\
\hline 8 & $1.44 \mathrm{E}+08$ & $8 q 24.3$ & 2978 & GML, CYP11B1, CYP11B2 & 0.001282 \\
\hline 8 & 75824814 & $8 \mathrm{q} 21.11$ & 4208 & PI15 & 0.002815 \\
\hline 8 & $1.45 \mathrm{E}+08$ & $8 q 24.3$ & 1284 & ZC3H3, GSDMDC1, C8orf73, NAPRT1, EEF1D & 0.003108 \\
\hline 8 & $1.18 \mathrm{E}+08$ & $8 \mathrm{q} 24.11$ & 4296 & & 0.003307 \\
\hline 8 & 72080962 & $8 \mathrm{q} 13.3$ & 5059 & & 0.00559 \\
\hline 8 & 76752360 & $8 \mathrm{q} 21.11$ & 4850 & & 0.00832 \\
\hline 8 & 88062342 & $8 \mathrm{q} 21.3$ & 5174 & CNBD1 & 0.008437 \\
\hline 8 & 90966600 & $8 \mathrm{q} 21.3$ & 2711 & C8orf1, NBN & 0.008991 \\
\hline 9 & $1.08 \mathrm{E}+08$ & $9 \mathrm{q} 31.2$ & 4892 & & 0.004541 \\
\hline 12 & $1.03 \mathrm{E}+08$ & $12 \mathrm{q} 23.3$ & 2790 & NT5DC3 & 0.00217 \\
\hline 13 & 31754912 & $13 q 13.1$ & 148 & FRY, BRCA2, IFIT1P & 0.007934 \\
\hline 14 & 63953132 & $14 q 23.2-$ & 1066 & MTHFD1, AKAP5, ZBTB25 & 0.002416 \\
\hline 14 & 52954585 & $14 q 22.1$ & 4506 & & 0.00685 \\
\hline 14 & 68799746 & $14 \mathrm{q} 24.1$ & 4082 & GALNTL1 & 0.007369 \\
\hline 14 & 76963463 & $14 \mathrm{q} 24.3$ & 1546 & C14orf133, AHSA1, THSD3, SPTLC2 & 0.007369 \\
\hline 15 & 89157227 & $15 \mathrm{q} 26.1$ & 2434 & BLM, FURIN, FES, MAN2A2 & 0.007798 \\
\hline 20 & 22946651 & 20p11.21 & 2292 & SSTR4, THBD, CD93 & 0.00474 \\
\hline 21 & 35144508 & $21 q 22.12$ & 2331 & RUNX1 & 0.002614 \\
\hline $\mathrm{X}$ & 22016295 & $\mathrm{Xp} 22.11$ & 4307 & PHEX, ZNF645 & 0.003979 \\
\hline \multicolumn{6}{|l|}{$\begin{array}{l}\text { DNA copy } \\
\text { number loss }\end{array}$} \\
\hline 2 & $2.06 \mathrm{E}+08$ & $2 q 33.3$ & 825 & ALS2CR19 & 0.002614 \\
\hline 2 & $1.32 \mathrm{E}+08$ & $2 q 21.1$ & 2745 & ARHGEF4, PLEKHB2 & 0.006666 \\
\hline 2 & $2.10 \mathrm{E}+08$ & $2 q 34$ & 1526 & MAP2 & 0.007483 \\
\hline 3 & 14148375 & $3 \mathrm{p} 25.1$ & 2562 & TMEM43, XPC, LSM3 & 0.000792 \\
\hline 3 & 71826780 & $3 p 13$ & 900 & EIF4E3, GPR27, PROK2 & 0.002771 \\
\hline 3 & 74941458 & $3 \mathrm{p} 12.3$ & 4309 & & 0.002771 \\
\hline 3 & $1.39 \mathrm{E}+08$ & $3 q 22.3$ & 418 & NPM1P17 & 0.004333 \\
\hline 3 & 36923131 & $3 \mathrm{p} 22.3$ & 3024 & EPM2AIP1, MLH1 & 0.004417 \\
\hline 3 & 63273432 & 3 p14.2 & 1005 & SYNPR & 0.005318 \\
\hline 3 & 26969537 & $3 \mathrm{p} 24.2$ & 4929 & & 0.005992 \\
\hline 3 & 64300928 & $3 p 14.1$ & 348 & & 0.006545 \\
\hline 3 & $1.87 \mathrm{E}+08$ & $3 q 27.2$ & 2819 & ETV5 & 0.00685 \\
\hline 3 & 25613645 & $3 \mathrm{p} 24.2$ & 2268 & RARB, TOP2B & 0.00936 \\
\hline 3 & 44861037 & $3 \mathrm{p} 21.31$ & 5340 & KIF15, TMEM42, TGM4, ZDHHC3 & 0.009527 \\
\hline 3 & 61112371 & $3 p 14.2$ & 2781 & FHIT & 0.009527 \\
\hline 3 & 833434 & $3 \mathrm{p} 26.3$ & 5701 & & 0.009665 \\
\hline 4 & 57604869 & $4 q 12$ & 656 & REST, C4orf14, POLR2B, IGFBP7 & 0.007076 \\
\hline 4 & $1.66 \mathrm{E}+08$ & $4 \mathrm{q} 32.3$ & 4287 & & 0.008078 \\
\hline 5 & $1.26 \mathrm{E}+08$ & $5 \mathrm{q} 23.2$ & 4736 & LMNB1, MARCH3 & 0.00767 \\
\hline 5 & $1.25 \mathrm{E}+08$ & $5 q 23.2$ & 4761 & & 0.007819 \\
\hline 6 & 58452627 & $6 p 11.2$ & 998 & & 0.006476 \\
\hline 8 & 6562724 & $8 \mathrm{p} 23.1$ & 4738 & AGPAT5 & 0.004137 \\
\hline 8 & 36251863 & $8 p 12$ & 4919 & & 0.005148 \\
\hline 8 & 649574 & $8 \mathrm{p} 23.3$ & 5579 & ERICH1, C8orf68 & 0.007967 \\
\hline 8 & 250609 & $8 p 23.3$ & 670 & $\mathrm{FBXO} 25$ & 0.009945 \\
\hline 9 & 414664 & $9 \mathrm{p} 24.3$ & 5566 & DOCK8, ANKRD15 & 0.002083 \\
\hline 9 & 14314735 & $9 \mathrm{p} 22.3$ & 5623 & & 0.004436 \\
\hline
\end{tabular}


Table VI. Continued.

\begin{tabular}{|c|c|c|c|c|c|}
\hline Chromosome & BAC-start & Locus & ID & Locating genes & $\mathrm{P}$-value \\
\hline \multicolumn{6}{|l|}{$\begin{array}{l}\text { DNA copy } \\
\text { number loss }\end{array}$} \\
\hline 9 & 15666106 & $9 p 22.3$ & 4639 & C9orf93 & 0.006505 \\
\hline 9 & 288712 & $9 \mathrm{p} 24.3$ & 2768 & DOCK8 & 0.007162 \\
\hline 9 & 502548 & 9 p24.3 & 5569 & ANKRD15 & 0.007419 \\
\hline 10 & 543426 & $10 \mathrm{p} 15.3$ & 230 & DIP2C & 0.000366 \\
\hline 10 & 183360 & $10 \mathrm{p} 15.3$ & 2532 & ZMYND11 & 0.001822 \\
\hline 10 & 185862 & $10 \mathrm{p} 15.3$ & 2166 & ZMYND11 & 0.005187 \\
\hline 10 & 854943 & $10 \mathrm{p} 15.3$ & 5553 & LARP5 & 0.006853 \\
\hline 12 & 66758603 & $12 q 15$ & 2084 & IFNG & 0.000903 \\
\hline 12 & 38170799 & $12 q 12$ & 4224 & $\mathrm{ABCD} 2$ & 0.006666 \\
\hline 15 & 19051540 & $15 q 11.2$ & 5162 & & 0.002692 \\
\hline 15 & 37896537 & $15 q 14-15 q 15.1$ & 1056 & GPR176 & 0.006096 \\
\hline 17 & 10455531 & $17 \mathrm{p} 13.1$ & 1536 & MYH3, SCO1, C17orf48 & 0.000563 \\
\hline 17 & 3811567 & $17 \mathrm{p} 13.2$ & 1210 & ATP2A3, ZZEF1 & 0.00282 \\
\hline 19 & 21158366 & $19 \mathrm{p} 12$ & 2301 & ZNF431 & 0.007369 \\
\hline 20 & 3477726 & 20p13 & 1566 & ATRN & 0.008263 \\
\hline 20 & 4970392 & 20p13-20p12.3 & 2878 & C20orf30, PCNA, CDS2 & 0.009205 \\
\hline 22 & 48278710 & $22 q 13.33$ & 930 & & 0.005187 \\
\hline $\mathrm{Y}$ & 1805965 & Yp11.31 & 5180 & & 0.006476 \\
\hline
\end{tabular}

information for patients with $\mathrm{CRC}$, such as the presence of lymph node metastasis, the clinical stage, and lymphovascular and venous invasion, are predictors of prognosis and indicators of the likelihood of a response to treatment. In the present study, a-CGH yielded a more statistically meticulous analysis than previous chromosomal $\mathrm{CGH}$.

Our previous CGH analysis showed that a gain of $8 \mathrm{q} 24$ was the most significant factor related to lymph node metastasis and advanced tumor stage in CRCs $(7,8)$. Since advanced stage tumors are usually accompanied by lymph node metastasis, there were many overlapping DCNAs that were associated with lymph node metastasis and an advanced tumor stage in CRCs. However, elevated tumor stage alone was significantly associated with an increased frequency of a gain of $\mathrm{X}$ and chromosome 15 in the present study. Other factors that were determinants of an advanced tumor stage that were separate from lymph node metastasis were liver metastasis and peritoneal metastasis. However, since the number of these cases was limited in comparison to those with lymph node metastasis, the details could not be determined.

The 8q24 locus is frequently amplified not only in colorectal cancer but also in other types of cancer, e.g., breast cancer (10) and prostate cancer (11). A number of reports have speculated that aberrations of $8 \mathrm{q} 24$ are associated with malignant transformation of a cell, and the CMYC gene, which is located at $8 \mathrm{q} 24$, has been suggested as a dominant candidate gene for carcinogenesis $(7,8,10,11)$. However, since numerous genes other than CMYC are included in this region, multiple genes located at 8q24 may take part in the carcinogenesis.

In the present study, the CRC patients with loss of $20 \mathrm{p} 12.2$ demonstrated a high frequency of lymph node metastasis. The JAG1 gene is located at 20p12.2, and the NOTCH1 gene is at 9q34.4. Although these loci are associated with ARAJIRU syndrome, elevated expression of JAG1 and NOTCH1 was also reported as a marker of poor prognosis in breast cancer $(12,13)$. The same association has been reported in CRCs (14). Furthermore, JAG1 mRNA has been shown significantly increased in most familial adenomatous polyposis (FAP) adenomas in comparison to that of normal intestinal tissue (15). Table IV demonstrates that a loss of 20p12.2 (clone 768), on which JAG1 is located, has a significant correlation with the lymph node metastasis of CRC $(\mathrm{p}<0.01)$. Gain of $8 \mathrm{q} 24.3$ and loss of 20p12.2 were independent factors for lymph node metastasis. All 7 CRCs that had either 8q24.3 gain or 20p12.2 loss had lymph node metastasis. Among $32 \mathrm{CRCs}$ with either aberration, 26 tumors demonstrated lymph node metastasis. Furthermore, CRCs with lymphovascular invasion showed an increase of $8 \mathrm{q} 2$ gain in addition to $10 \mathrm{q} 21$ loss, which is reported to be associated with Alzheimer's disease (16).

CRCs with venous invasion were associated with an increased frequency of losses of various BAC clones. For example, losses of $12 \mathrm{q} 15$ and 17 p13.1 encoding IFNG and p53, respectively, were both associated with venous invasion. Furthermore, the results showed a high frequency of losses of the cadherin-related genes, for example, the $\beta$-catenin domain was associated with lymph node metastasis, and the $\alpha-3$ catenin domain with lymphovascular invasion $(14,16)$. Therefore, metastatic colorectal cancer might not express either cadherin or catenin proteins, which may result in exfoliated cancer cell metastases.

In conclusion, the present study examined the relationship between the clinicopathological features of CRC and DCNAs: a gain of $8 q 24.3$ and losses of 9q33.1 and 20p12.2 were associated with lymph node metastasis, gain of $8 \mathrm{q} 24.3$ and loss of 9q33.1 with disease stage, gain of $8 \mathrm{q} 21.11$ and loss of $10 \mathrm{q} 21.3$ with lymphovascular invasion and losses of $3 \mathrm{p} 25.1$, 
$10 \mathrm{p} 15.3,12 \mathrm{q} 15$, and $17 \mathrm{p} 13.1$ for venous invasion. These aberrations can be regarded as genomic biomarkers to predict the clinical outcome of the patients with $\mathrm{CRC}$, and are expected to serve to individualize the treatment of CRC patients. Further clarification of the genomic and clinical correlations is expected to provide more useful genomic biomarkers for clinical management of the patients with CRC.

\section{References}

1. Hamilton SR, Vogelstein B, Kudo S, Riboi E, Nakamura S, Hainaut P, Rubio CA, Sobin LH, Fogt F, Winawer SJ, Goldgar DE and Jass JR: Carcinoma of the colon and rectum. In: World Health Organization Classification of Tumors: Pathology and Genetics of Tumors of the Digestive System. Hamilton SR and Aaltonen LA (eds). IARC Press, Lyon, pp105-119, 2000.

2. Fearon ER and B Vogelstein: A genetic model for colorectal tumorigenesis. Cell 61: 759-767, 1990.

3. Lengauer C, Kinzler KW and Vogelstein B: Genetic instability in colorectal cancers. Nature 386: 623-627, 1997.

4. Pinkel D, Segraves R, Sudar D, Clark S, Poole I, Kowbel D, Collins C, Kuo WL, Chen C, Zhai Y, Dairkee SH, Ljung BM, Gray JW and Albertson DG: High resolution analysis of DNA copy number variation using comparative genomic hybridization to microarrays. Nat Genet 20: 207-211, 1998.

5. Veltman JA, Fridlyand J, Pejavar S, Olshen AB, Korkola JE, DeVries S, Carroll P, Kuo WL, Pinkel D, Albertson D, CordonCardo C, Jain AN and Waldman FM: Array-based comparative genomic hybridization for genome-wide screening of DNA copy number in bladder tumors. Cancer Res 63: 2872-2880, 2003.

6. Lengauer C, Kinzler KW and Vogelstein B: Genetic instabilities in human cancers. Nature 396: 643-649, 1998.

7. Liu XP, Kawauchi S, Oga A, Sato T, Ikemoto K, Ikeda E and Sasaki K: Chromosomal aberrations detected by comparative genomic hybridization predict outcome in patients with colorectal carcinoma. Oncol Rep 17: 261-267, 2007.

8. Nakao M, Kawauchi S, Furuya T, Uchiyama T, Adachi J, Okada T, Ikemoto K, Oga A and Sasaki K: Identification of DNA copy number aberrations associated with metastases of colorectal cancer using array CGH profiles. Cancer Genet Cytogenet 188: 70-76, 2009.
9. UICC TNM Classification of Malignant Tumours. LH Sobin and Wittekind CH (eds). 6th edition. Wiley-Liss, Oxford, 2002.

10. Naylor TL, Greshock J, Wang Y, Colligon T, Yu QC, Clemmer V, Zaks TZ and Weber BL: High resolution genomic analysis of sporadic breast cancer using array-based comparative genomic hybridization. Breast Cancer Res 7: 1186-1198, 2005.

11. van Duin $M$, van Marion R, Vissers $K$, Watson JE, van Weerden WM, Schroder FH, Hop WC, van der Kwast TH, Collins C and van Dekken H: High-resolution array comparative genomic hybridization of chromosome arm $8 \mathrm{q}$ : evaluation of genetic progression markers for prostate cancer. Genes Chromosomes Cancer 44: 438-449, 2005.

12. Reedijk M, Odorcic S, Chang L, Zhang H, Miller N, McCready DR, Lockwood G and Egan SE: High-level coexpression of JAG1 and NOTCH1 is observed in human breast cancer and is associated with poor overall survival. Cancer Res 65: 8530-8537, 2005.

13. Reedijk M, Pinnaduwage D, Dickson BC, Mulligan AM, Zhang H, Bull SB, O'Malley FP, Egan SE and Andrulis IL: JAG1 expression is associated with a basal phenotype and recurrence in lymph node-negative breast cancer. Breast Cancer Res Treat 111: 439-448, 2008.

14. Rodilla V, Villanueva A, Obrador-Hevia A, Robert-Moreno A, Fernandez-Majada V, Grilli A, Lopez-Bigas N, Bellora N, Alba MM, Torres F, Dunach M, Sanjuan X, Gonzalez S, Gridley T, Capella G, Bigas A and Espinosa L: Jagged1 is the pathological link between Wnt and Notch pathways in colorectal cancer. Proc Natl Acad Sci USA 106: 6315-6320, 2009.

15. Robert-Moreno A, Guiu J, Ruiz-Herguido C, Lopez ME, Ingles- Esteve J, Riera L, Tipping A, Enver T, Dzierzak E, Gridley T, Espinosa L and Bigas A: Impaired embryonic haematopoiesis yet normal arterial development in the absence of the Notch ligand Jagged1. EMBO J 27: 1886-1895, 2008.

16. Miyashita A, Arai $\mathrm{H}$, Asada $\mathrm{T}$, Imagawa $\mathrm{M}$, Matsubara $\mathrm{E}$, Shoji M, Higuchi S, Urakami K, Kakita A, Takahashi H, Toyabe S, Akazawa K, Kanazawa I, Ihara Y and Kuwano R: Genetic association of CTNNA3 with late-onset Alzheimer's disease in females. Hum Mol Genet 16: 2854-2869, 2007. 